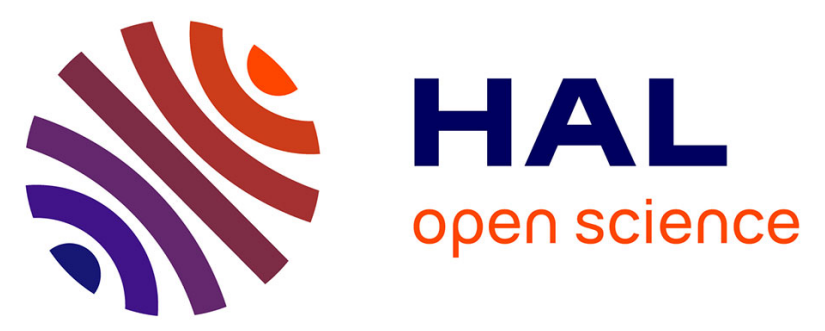

\title{
Time to and differential time to blood culture positivity for assessing catheter-related yeast fungaemia: A longitudinal, 7-year study in a single university hospital
}

Maud Gits-Muselli, Stephane Villiers, Samia Hamane, Béatrice Berçot, Jean-Luc Donay, Blandine Denis, Nicolas Guigue, Alexandre Alanio, Stéphane Bretagne

\section{To cite this version:}

Maud Gits-Muselli, Stephane Villiers, Samia Hamane, Béatrice Berçot, Jean-Luc Donay, et al.. Time to and differential time to blood culture positivity for assessing catheter-related yeast fungaemia: A longitudinal, 7-year study in a single university hospital. Mycoses, 2019, 10.1111/myc.13024 . pasteur-02418220

\section{HAL Id: pasteur-02418220}

\section{https://hal-pasteur.archives-ouvertes.fr/pasteur-02418220}

Submitted on 18 Dec 2019

HAL is a multi-disciplinary open access archive for the deposit and dissemination of scientific research documents, whether they are published or not. The documents may come from teaching and research institutions in France or abroad, or from public or private research centers.
L'archive ouverte pluridisciplinaire HAL, est destinée au dépôt et à la diffusion de documents scientifiques de niveau recherche, publiés ou non, émanant des établissements d'enseignement et de recherche français ou étrangers, des laboratoires publics ou privés.

\section{(1) $\$(0)$}

Distributed under a Creative Commons Attribution - NonCommercial - ShareAlikel 4.0 
DR MAUD GITS-MUSELLI (Orcid ID : 0000-0002-0470-6866)

DR BLANDINE DENIS (Orcid ID : 0000-0003-2101-2601)

Article type : Original Article

\section{MYC-OA-2019-106 revised 1}

\section{Time to and differential time to blood culture positivity for assessing} catheter-related yeast fungaemia: A longitudinal, 7-year study in a single university hospital

Running title: TTP, DTTP and CVC- related yeast fungaemia

Maud Gits-Musellia ${ }^{\mathrm{ab}, \mathrm{c}^{*}}$, Stéphane Villiers ${ }^{\mathrm{d}}$, Samia Hamane ${ }^{\mathrm{a}}$, Béatrice Bercote,f ${ }^{\mathrm{e}}$, Jean-Luc Donay ${ }^{\mathrm{e}}$, Blandine Denis ${ }^{\mathrm{g}}$, Nicolas Guigue ${ }^{\mathrm{a}}$, Alexandre Alanio ${ }^{\mathrm{a}, \mathrm{b}, \mathrm{c}}$, and Stéphane Bretagne $\mathrm{e}^{\mathrm{a}, \mathrm{b}, \mathrm{c}}$

a Parasitology-Mycology Laboratory, Lariboisière Saint-Louis Fernand Widal Hospital, Assistance Publique-Hôpitaux de Paris (AP-HP)

b Paris-Diderot, Sorbonne Paris Cité University

c Institut Pasteur, Molecular Mycology Unit, Reference National Center of Invasive Mycoses and Antifungals, CNRS UMR2000, Paris75015, France

d Anesthesiology Department, Lariboisière Saint-Louis Fernand Widal Hospital, Assistance Publique-Hôpitaux de Paris (AP-HP)

e Microbiology Department, Lariboisière Saint-Louis Fernand Widal Hospital, Assistance Publique-Hôpitaux de Paris (AP-HP)

f Paris-Diderot, Sorbonne Paris Cité University, IAME UMR-1137, Paris

g Tropical and Infectious Diseases Department, Lariboisière Saint-Louis Fernand Widal Hospital, Assistance Publique-Hôpitaux de Paris (AP-HP)

This article has been accepted for publication and undergone full peer review but has not been through the copyediting, typesetting, pagination and proofreading process, which may lead to differences between this version and the Version of Record. Please cite this article as doi: 10.1111/MYC.13024 
*Corresponding author : Laboratoire de Parasitologie et de Mycologie, Hôpital Saint Louis, 1 avenue Claude Vellefaux, 75475 Paris, France

Phone: +331 42499502 ; Fax: +331 424948 03; Email: maud.gits-muselli@aphp.fr

Presented in part: 27th ECCMID, April 2017 Vienna, PO n 2006

\section{Acknowledgments}

We thank Pr Françoise Dromer for her valuable comments on the manuscript and Raphaël Goudinoux for providing the CVCs type data.

\section{Statement of Authors contributions}

MGM, SV, SH and NG participated in data analysis.

MGM, SB and AA wrote the manuscript.

$\mathrm{BB}, \mathrm{JLD}$ and $\mathrm{BD}$ made practical comment on the results and statistics

All the authors read the manuscript and agreed with the final version.

Financial support: This work was performed with the results obtained through our routine diagnosis process and did not benefit from a specific founding.

Disclaimers: No conflict of interest to be declared for the present study. 


\section{Abstract:}

Background: Time to positivity (TTP) and differential time to positivity (DTTP) between central and peripheral blood cultures are commonly used for bacteraemia to evaluate the likelihood of central venous catheter (CVC) related bloodstream infection. Few studies have addressed these approaches to yeast fungaemia.

Objectives: This study aimed to evaluate TTP and DTTP to assess CVC-related yeast fungaemia (CVC-RYF).

Patients/Methods: We retrospectively analysed the results from 105 adult patients with incident fungaemia, with CVC removed and cultured, collected from 2010 to 2017. The bottles were incubated in a BioMérieux BacT/ALERT 3D and kept for at least 5 days.

Results: Of the 105 patients included, most were oncology patients $(85.7 \%)$ and had of long-term CVC (79.6\%); 32 (30.5\%) had a culture positive CVC (defined as CVC-RYF) with the same species as in blood culture and $69.5 \%$ had culture negative CVC (defined as non-CVC-RYF, NCVC-RYF). Candida albicans represented $46 \%$ of the episodes. The median TTP was statistically different between CVC-RYF or NCVC-RYF [16.8h interquartile range (IQR) [9.728.6] vs. 29.4h [IQR 20.7-41.3]; $\mathrm{p}=0.001]$. A TTP $<10 \mathrm{~h}$ had the best positive likelihood ratio (21.5) for CVC-RYF, although the sensitivity was only $28 \%$. DTTP was available for 52 patients. A DTTP $>5 \mathrm{~h}$ had a sensitivity of $100 \%$ and a specificity of $71 \%$ for CVC-RYF. Conclusions:

Since the median TTP was $17 \mathrm{~h}$ and the most performing DTTP $>5 \mathrm{~h}$, these delays are too long to take a decision in the same operational day. More rapid methods for detecting infected catheters should be tested to avoid unnecessary CVC withdrawal.

Keywords: Blood cultures, central venous catheter, yeast fungaemia, candidaemia, diagnosis, time to positivity, differential time to positivity 


\section{INTRODUCTION}

Central venous catheters (CVCs) significantly increase the risk of candidaemia, and inversely, candidaemia is often associated with intravascular catheters. ${ }^{1}$ Indeed, the increased incidence in candidaemia has paralleled the increased use in a variety of indwelling catheters. CVCs are present in at least $80 \%$ of cancer patients with candidaemia, for which the source of infection can be clinically related to the catheter. ${ }^{2,3}$ As a consequence, most guidelines for candidaemia management recommend removal of existing intravascular catheters, if feasible. ${ }^{4-7}$ Although the recommendation is strong, the evidence is of low-quality, and various authors have recommended a re-consideration of CVC removal on an individual basis. ${ }^{4-8}$ Practically, one must consider the possible deleterious consequence of CVC removal, especially in patients who require long-term CVC for cancer treatment or parenteral nutrition. This scenario is even more complicated to manage when the catheter's infection is not subsequently confirmed.

To incriminate a CVC as a source of bacteremia, time to positivity (TTP) and differential time to positivity (DTTP) have been extensively studied and are routinely used for decision making. ${ }^{9-11}$ Few similar analyses have been performed for CVC-related candidaemia but with controversial results and no definitive thresholds. ${ }^{2,12,13}$ In parallel, TTP has also been evaluated to identify yeast species as soon as the blood culture is positive. ${ }^{14-17} \mathrm{We}$, therefore, used our comprehensive database on yeast-positive blood culture episodes collected over 7 years in our institution to analyse the usefulness of TTP and DTTP to predict CVC-related yeast fungaemia (CVC-RYF).

\section{MATERIALS AND METHODS}

\section{Study design and data collection}

We enrolled all $\geq 15$-year-old patients for whom at least one blood culture $(\mathrm{BC})$ bottle was positive for yeast between October $1^{\text {st, }} 2010$ and September $30^{\text {th, }} 2017$ through the records of the Department of Microbiology of Saint-Louis Hospital. This hospital is a 550-bed tertiary university hospital located in Paris, France, with more than two-thirds of its activity dedicated to haematology or oncology patients. Burn patients were excluded as these were analysed separately because all the removed catheter tips were culture-negative for Candida spp. suggesting that CVC is not the source of candidaemia whereas candidaemia was always concomitant with Candida carriage of the skin. ${ }^{18}$

The clinical data collected were age, gender, underlying conditions, hospitalization in 
intensive care unit (ICU) at the time of positive $\mathrm{BC}$, antifungal preexposure defined as administration of systemic antifungal drugs for at least 5 days within 30 days prior to fungaemia (whatever dosage and duration), and outcome at day 30 after the first positive BC as described elsewhere. ${ }^{19}$ Because of the retrospective design of the study and the multiple regimens of antifungal drugs prescribed in haematology, it was not possible to reliably collect more data on type, dose, and duration of the antifungal drugs.

The type of CVCs, its duration, and the reason for removing it were collected from the Anesthesiology Department. The CVCs were divided into short-term (mainly from ICU) and longterm CVC.

\section{Microbiological methods}

Nurses were instructed to draw $10 \mathrm{ml}$ of blood into each aerobe and anaerobe bottles and to send the inoculated bottles to the microbiology laboratory without delay. Bottles were incubated (BioMérieux BacT/ALERT 3D, Marcy l'Etoile, France) for at least 5 days or until positivity. When the $\mathrm{BC}$ was detected as positive, and the Gram stain demonstrated the presence of yeasts, a

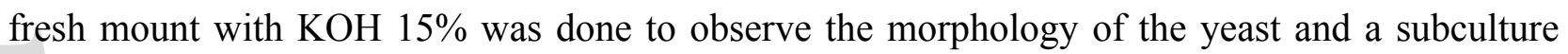
was performed in BBL CHROMagar Candida Medium plate (Hardy Laboratories, Santa Monica, CA, USA). Identification was confirmed using the API ID 32C (bioMérieux, Marcy l'Etoile, France) from October 2010 to the end of 2011, then the Biomerieux MALDI-TOF system (VITEK-MS) (bioMérieux, Marcy l'Etoile, France) from January 2013 to September 2017. For the uncommon fungal species [i.e. not for Candida albicans, C. glabrata, C. tropicalis, C. parapsilosis, C. krusei (syn. Pichia kudriavzevii), and Cryptococcus spp.], identification was confirmed by molecular analysis at the National Reference Centre for Invasive Mycoses and Antifungals at Institut Pasteur, Paris, France, as described elsewhere. ${ }^{19}$

After CVC removal, the catheter tip and/or the chamber of the implantable venous access port system were cultured using a quantitative method. ${ }^{20}$ Briefly, tip and/or chamber were immersed in $1 \mathrm{~mL}$ of sterile water and vortexed for $1 \mathrm{~min}$. Then, $50 \mu \mathrm{L}$ were plated on a blood and chocolate agar Petri dishes. The plate was then incubated at $35^{\circ} \mathrm{C}\left( \pm 2^{\circ} \mathrm{C}\right)$ with $5 \%( \pm 1 \%) \mathrm{CO}_{2}$ for 24h. CVC culture was considered negative (CVC-negative) if there was no growth observed after 5 days of culture. A CVC was considered positive (CVC-positive) if either the tip, the chamber, or both grew at least one yeast colony. 


\section{Definition of CVC-related yeast fungaemia}

The date of fungaemia was the date of blood sampling. Paired bottles were defined as respectively drawn through $\mathrm{CVC}$ and percutaneously within less than $30 \mathrm{~min}$. TTP is routinely recorded by the BacT/Alert system as the time recorded until growth detection after recording the $\mathrm{BC}$ bottle in the incubation cabinet. DTTP is defined as the difference in TPP recorded for paired bottles (DTTP = TTP percutaneously - TTP CVC). When multiple positive BC were available for a given patient, only the first was considered for TPP analysis and only the first paired bottles for DTTP, since this was the first information available for the clinicians.

Patients with CVC removed and cultured at the time of fungemia were divided into two groups: (i) CVC-related yeast fungemia (CVC-RYF) when the same yeast species was recovered from at least one $\mathrm{BC}$ and from the $\mathrm{CVC}$ culture; (ii) non-CVC-related yeast fungemia (NCVCRYF) when a yeast species was recovered from at least one $\mathrm{BC}$, and the CVC culture was negative or yielded a different species. ${ }^{12}$ This definition of CVC-RYF was adapted from the definition proposed by Bouza et al. except we considered for TTP calculation the patients with a positive BC drawn through CVC and not only the patient with a positive peripheral BC. ${ }^{12}$

\section{Statistical analysis}

Patients with CVC-RYF and NCVC-RYF were compared. Quantitative variables were reported as medians and interquartile ranges (IQR) or means \pm standard deviations. $\mathrm{Chi}^{2}$, Kruskal Wallis and Mann-Whitney tests were used for quantitative variables (TTP and DTTP). The Area Under a Receiver Operating Characteristic (ROC) Curve (AUC) was calculated to detect the best cut-off values for predicting CVC-RYF using TTP or DDTP values. A p-value $<0.05$ was considered significant. Prism 7 (GraphPad Software, La Jolla, CA, USA) was used for statistical analyses and graphs.

\section{Ethics statement}

The present study is a retrospective analysis of the data collected as part of our usual procedure for standard diagnostics with no additional sampling for the patients and no impact on management. This study was registered with our hospital review committee (reference number: 2018000000077). Because this was a retrospective observational study, it does not require the application for specific informed consent according to French law. 


\section{RESULTS}

\section{Selection of patients}

Over the 7 years, 249 episodes of fungaemia were recorded in 240 patients. The presence of CVC was noted in 203/240 (85\%) patients. The patients with CVC were more often oncology patients $(\mathrm{p}=0.01$, Supplemental Table S1). We excluded 98 patients because of: lack of CVC culture $(n=65)$, lack of BC through the CVC $(n=24)$, co-infection with bacteria $(n=17)$ or mixed yeasts infection $(\mathrm{n}=3$ ) (Figure 1). Finally, a total of 105 patients with CVC removed and cultured were selected for further analysis (Supplemental Table S1) The 98 patients excluded were not statistically different except that they had a less frequent antifungal pre-exposure $(p=0.01)$ than the selected patients. No recurrent episode for these 105 remaining patients was observed (Supplemental Table S1).

\section{Yeast identification and patient characteristics (Table 1)}

The 105 patients were divided into NCVC-RYF $(\mathrm{n}=73,69.5 \%)$ and CVC-RYF $(\mathrm{n}=32$, $30.5 \%$ ) based on the CVC culture results. The only significant difference between the two groups was the percentage of negative $\mathrm{CVC}$ culture when a preexposure to antifungal drugs was recorded [NCVC-RYF 22/73 (30\%) vs. CVC-RYF 2/32 (6\%); $\mathrm{p}=0.007]$.

The main species involved were C. albicans (46\%), C. parapsilosis (19\%), C. glabrata (15\%), and C. tropicalis (9\%), with no statistical difference between NCVC-RYF and CVC-RYF. Only four non-Candida species were identified (Table 1), representing less than $4 \%(4 / 105)$ of the total. Out of the 32 culture-positive CVCs, 24/32 (75\%) grew $>10^{3} \mathrm{CFU} / \mathrm{mL}$ and 8/32 (25\%) grew $>10^{2} \mathrm{CFU} / \mathrm{mL}$ without difference in the species distribution or the CVC features (short-term vs. long-term $\mathrm{CVC}$ ) according to the number of colonies and were therefore not subsequently differentiated.

Most of the CVCs were long-term [82/105; 78\%; median duration time: 68 days [IQR: 22157], and were more frequent in onco-hematology patients. These long-term CVCs were including totally implantable venous access port system $(n=44)$, tunnelled cuffed catheter $(n=32)$, and peripheral insertion central catheter $(n=6)$. The long-term central venous access catheters were not more frequent in CVC-RYFs than in NCVC-RYFs (Table 1). In contrast, the 23 short-term CVCs were more frequent in ICU patients $(21 / 23$; $91.3 \%)$ with a median duration time of 10 days [IQR: 6-14]. The motive for removal and culture was always a suspicion of infection. Only 30.5\% (32/105) of the tested CVCs were culture-positive, with no statistical difference between long and 
short-term CVCs (data not shown). The median delay between the date of blood sampling of the first positive BC and the date of CVC removal was 3 days [IQR 2-3] and 2 [IQR 1-3] for NCVCRYFs and CVC-RYFs respectively $(\mathrm{p}=0.08)$.

\section{TTP according to CVC or non-CVC related yeast-fungaemia}

Considering all species, the median TTP of the first bottle collected from CVC was statistically shorter in the CVC-RYF group (16.8h [IQR 9.7-28.6]) compared to the NCVC-RYF group: median (29.4h [IQR 20.7-41.3], p = 0.0001, Figure 2). When analysing TTP regarding the Candida species, a significant shorter TPP was only observed in C. albicans with a median of 12.9h [10.0-22.7] vs. 32.3h [24.4-41.0]) for CVC-RYF and NCVC-RYF, respectively ( $\mathrm{p}=$ 0.0001, Figure 2). A trend towards shorter TTPs in the CVC-RYF vs. NCVC-RYF was observed for $C$. tropicalis $(\mathrm{p}=0.09)$ and $C$. glabrata $(\mathrm{p}=0.17)$. No difference was observed for $C$. parapsilosis $(\mathrm{p}=0.86)$. The TTP distribution was not statistically different (Kruskal Wallis test; $\mathrm{p}$ $=0.9$ ) between patients who died compared with the patients alive at 30 days (Table 1). The median TTP values were not statistically different $(p=0.9)$ when comparing NCVC-RYF patients with (median TTP $=32 \mathrm{~h}$ ) or without (median TTP $=31 \mathrm{~h}$ ) pre-exposition with antifungal agent.

Using TPP as a predictor of CVC-RYF, we obtained an AUC of 0.73 [Confidence Interval 95\% (CI95\%) 0.62 to $0.85, \mathrm{p}=0.0001$ ] when considering all yeast species (Figure 3 ). A threshold of $<10 \mathrm{~h}$ provided the best positive likelihood ratio (21.6), but the sensitivity was only $28.1 \%$ (Table 2). When restricting the analyses to C. albicans (Figure 3), the AUC was improved (0.84 [CI95\% 0.69 to 1.00], $\mathrm{p}=0.0002$ ). A threshold of $<15 \mathrm{~h}$ was associated with a high positive likelihood ratio (30.5), with a sensitivity of 61\% (Supplemental Table S2).

Among the 105 patients included in the study, 65 (62\%) had a peripheral positive BC. When restricted the TTP analysis to these patients, the rate of infected CVC increased from 30.5\% $(32 / 105)$ to $33.8 \%(22 / 65)$, which is not statistically significant, $p=0.6$. The median TTP was 11.75h [IQR 8.5-20.4] for CVC-RYF (vs. 16.8h [IQR 9.7-28.6] for the 105 patients) and 29.3h [IQR 22.7-43.2] (vs. 29.4h [IQR 20.7-41.3] for the 105 patients) for NCVC-RYF (p <0.0001). Out of the 40 patients with no positive peripheral BC recorded, $24(60 \%)$ had a peripheral negative $\mathrm{BC}$, and $16(40 \%)$ did not have any peripheral BC performed.

\section{DTTP according to CVC or non-CVC related yeast fungaemia}

DTTP was available for 52/105 (49.5\%) patients, including 32 NCVC-RYF and 20 CVC- 
RYF. The median DTTP was significantly higher for CVC-RYF (18.8h [IQR 15.0-22.7]) than for NCVC-RYF (-0.4h [IQR -5.1-4.8]) patients, $\mathrm{p}<0.0001)$. Calculation by species was not possible given the low number of positive pairs.

The AUC was 0.86 ([CI95\% 0.74 to 0.98], p <0.0001) for the diagnosis of CVC-RYF without difference between C. albicans and non-albicans Candida spp. (Figure 4). The sensitivity was $100 \%$ in the first $5 \mathrm{~h}$, which is translated in a null negative likelihood ratio, although the specificity did not exceed $71 \%$ and the positive likelihood ratio was never $>5$ (Table 3 ).

\section{DISCUSSION}

When analysing 105 patients with CVC withdrawn and cultured, and using a strict definition of infected CVC (i.e., positive or negative CVC culture), a TTP $<10 \mathrm{~h}$ had the best likelihood ratio (21.6), although the sensitivity was only $28.1 \%$ for identifying CVC-RYF. A DTTP between 1 and $5 \mathrm{~h}$ presented 100\% specificity but a low positive likelihood ratio (LOR $<4)$. Our observation stands for a population of oncology patients $(86 \%)$ with mainly long-term central venous access catheter (78\%). Candida albicans represented $46 \%$ of the episodes followed by $C$. parapsilosis (19\%). We nevertheless kept the term of fungaemia, acknowledging that only 4 were not Candida species.

Comparison of TTP results with previous reports is difficult because of numerous differences in the studies design, inclusion of children or only adults, different underlying diseases, single or multiple centres recruitment, number of long-term or short-term CVCs included, culture method used for testing CVC tips, variable definitions of CVC infection with probable criteria added, and various Candida spp. considered). Also, the small number of fungaemia and the even smaller number of CVC-RYF add to the heterogeneity. The number of analysed episodes of CVC-RYF rarely reach more than 100 [22 in ${ }^{14}, 64 \mathrm{in}^{2}, 108 \mathrm{in}^{12}$, and $\left.105 \mathrm{in}^{13}\right]$ compared to 105 in our study, which precludes subset analyses. Here the method used for CVC testing was different from the one used by other authors. ${ }^{2,12}$ Moreover, we analysed the TTP for patients with negative or no peripheral $\mathrm{BC}$ in contrast to Bouza et al. ${ }^{12}$ and Ben-Ami et al. ${ }^{2}$ who included only patients with at least one positive peripheral BC. When excluding patients with no positive peripheral BC, the TTP decreased to 11.75h [IQR 8.5-20.4] vs. 16.8h [IQR 9.7-28.6] for CVC-RYF. This probably results from the higher fungal load when both peripheral and central bottles are positive. However, to exclude patients without peripheral positive BC does not correspond to onco-haematology patients with long-term $\mathrm{CVC}$ for who peripheral $\mathrm{BC}$ is not 
obtained because of practical or comfort reasons. Additionally, there is no recommendation to consider peripheral and central yeast fungaemia differently. ${ }^{4-8}$

Taking all these limitations into account, our best TTP cut-off of $10 \mathrm{~h}$ is different from other proposals. Ben-Ami et al. suggest a TTP cut-off of $30 \mathrm{~h}$ (100\% sensitive and $51.4 \%$ specific) for CVC-related candidaemia with an AUC of 0.76, close to our observation (0.73). ${ }^{2}$ Bouza et al. ${ }^{12}$ found TTP to be significantly shorter in peripheral blood culture in adults with catheter-related candidaemia compared to adults with non CVC-related candidaemia (29.8 vs. 36.8h, p <0.03), which we confirmed even if the figures were slightly different (16.8 vs. 29.4h, $\mathrm{p}=0.001)$, and the best TTP cut-off for the prediction of catheter-related candidaemia in adults to be $<55 \mathrm{~h} .{ }^{12}$ Only two patients in that study had long-term CVCs (tunnelled-CVC). However, even the 10h cut-off that we proposed is too long to be clinically relevant when the goal is withdrawing the CVC as soon as possible. Indeed, the median TTP of the first bottle collected through the CVC was around $17 \mathrm{~h}$ in the present study, corresponding to a median of two operational days for catheter removal after blood sampling (Figure 2). Waiting further could be deleterious for the patients. ${ }^{23}$ When restricting to $C$. albicans, the AUC improved as the positive likelihood ratio for TTP with the threshold of $<15 \mathrm{~h}$, but this is a formal observation as long as the species is identified at least $24 \mathrm{~h}$ after BC positivity. Species identification could be achieved much faster using PCR-based identification tests, ${ }^{24}$ matrix-assisted laser desorption ionization time-of-flight mass spectrometry, ${ }^{25}$ or peptide nucleic acid fluorescence in situ hybridization. ${ }^{26}$

TTP can, however, be used for other purposes than diagnosing CVC-RYF. For instance, shorter TTPs was shown associated with mortality. ${ }^{27}$ In contrast, other authors found an association between long TTP and mortality. ${ }^{28}$ We did not observe any significant difference between the median TTP and the global mortality at day 30 (here 30\%), acknowledging that underlying diseases and patient management could prevail in the crude mortality over infected CVC. While it has been previously associated with the number of positive $\mathrm{BCs},{ }^{12}$ we did not find such an association.

TTP can also be used for presumptive species identification since $C$. glabrata growth rate is lower than that of other Candida spp. and is known for its decreased susceptibility to azoles. ${ }^{15-17}$ However, the current recommendations for the initial treatment of candidaemia is candin whatever the species is, ${ }^{4-6}$ which limits the clinical utility of TPP for identification.

For DTTP, Park et al. found an overall sensitivity and specificity of a DTTP of $>2 \mathrm{~h}$ for diagnosing CVC-RYF of $85 \%$ (CI95\%: $74 \%$ to $93 \%$ ) and $82 \%$ (CI9586\% 66\% to 92\%), 
respectively, when studying 99 pairs, 47 with definite, 14 with probable, and 38 with non CVCrelated candidaemia. ${ }^{12}$ The authors concluded that a DTTP of $>2 \mathrm{~h}$ was the optimal cut-off, except for CVC infection caused by C. glabrata. Other authors found this threshold accepted for bacteraemia would have a sensitivity of $94 \%$ but a specificity of $40 \% .{ }^{11}$ We did not observe major differences between 2 and $5 \mathrm{~h}$ for the DTTP performance, with specificity not exceeding $71 \%$ and positive likelihood ratio $<4$. An analysis by species was not performed because of the small number of pairs for each Candida species.

A noticeable point is the low yield of positive catheter culture. Only 30\% (32/105) of tested CVCs were positive in culture with the method used. ${ }^{20}$ In the literature, the rate of definite infected CVCs is variable: from $20.3 \%^{2}$ to $44.8 \%{ }^{13}$ or $56 \%{ }^{12}$. Part of these differences could come from the methods used, either semiquantitative (with a threshold of $>15 \mathrm{CFU}$ per catheter segment), ${ }^{22}$ or quantitative ( $>10^{2}$ CFU per catheter segment), ${ }^{20}$ although both are equivalent for bacteria $^{29}$ and knowing that both are usually accepted. ${ }^{9}$ Despite these differences, these observations suggest that CVCs could not be the main source of infection, even though they are present in $>80 \%$ of patients with candidaemia. On the other hand, some authors even suggest that a positive culture tip is not always linked to candidemia, ${ }^{30}$ which can interfere with the definition of CVC-RYF. If the CVC is mostly not the source of infection in the cases categorized as CVC$\mathrm{RYF}$, this could explain why the patients' survival is not impacted by CVC removal. ${ }^{8}$ Another explanation for the lack of benefit of CVC removal could be that dissemination has already occurred when the CVC is withdrawn. This raises the question of systematic early removal of $\mathrm{CVC}$ in the management of candidaemia since some authors did not report differences, ${ }^{8}$ knowing that patients who cannot tolerate CVC removal have often a worse prognosis. Nevertheless, CVC removal has to be evaluated on an individual basis, knowing that the consensus recommendation is CVC withdrawal if possible as soon as a BC is positive for Candida species. ${ }^{6,9,21}$ In our study, the removal of long-term CVCs was scheduled by the anaesthesiologist as soon as possible after the $\mathrm{BC}$ result in considering the availability of a surgery room for this removal.

The low yield of CVC culture can also be due to the consequence of previous antifungal drugs prescription, and we indeed observed a higher percentage of antifungal drugs preexposure in the group of culture-negative CVC $(p=0.007)$. This is obviously an important factor to consider when antifungal prophylaxis is increasing in haematology patients. However, the delay between the day of sampling of the first positive BC and CVC removal was short (median 2-3 days) and not statistically different between NCVC-RYF and CVC-RYF suggesting that an antifungal 
treatment between the clinical suspicion and the removal of the CVC may not impact the CVC culture. The low yield of CVC culture could be also due to non optimal culture method. For instance, the length of sonication is rarely controled. In this study, positive CVC culture yielded yeast CFU count above $10^{2} \mathrm{CFU} / \mathrm{mL}$ and was then strongly suggestive of CVC-RYF. ${ }^{31,32}$ Lower tresholds could be considered.

We must acknowledge some limitations in our study. The volume of blood collected was not controlled, whereas it is well known that the volume collected from CVC is usually higher than via peripheral venipuncture. ${ }^{33}$ The time between blood sampling and $\mathrm{BC}$ loading into the incubator was not controlled, knowing that delays tend to shorten TTP. ${ }^{34}$ The population studied was mostly $(86 \%)$ oncology patients thereby restricting our findings to this population. One can also argue the choice of analysing the first BC when multiple BCs were available instead of the BC with the shortest TTP. ${ }^{12}$ Our choice was clearly to look for a means to provide, as quickly as possible, information useful for the clinicians and to stick to routine practice.

In conclusion, a TTP $<10 \mathrm{~h}$ and a DTTP between 2 and $5 \mathrm{~h}$ could be relevant for incriminating a CVC during yeast fungaemia in oncology patients. However, even the shortest DTTP of $2 \mathrm{~h}$ as proposed by others ${ }^{13}$ means waiting longer than the already prolonged incubation time required for a BC to become positive for Candida spp. with the potential risk of maintaining an infected CVC. Clearly, rapid molecular-based methods are needed to speed the detection process $^{35}$ and to safely reduce the unnecessary removal of uninfected CVC, acknowledging that these new methods should be evaluated in real-world clinical settings. ${ }^{36}$ 


\section{References}

1. Ramage G, Mowat E, Jones B, Williams C, Lopez-Ribot J. Our current understanding of fungal biofilms. Crit Rev Microbiol. 2009;35(4):340-355. doi:10.3109/10408410903241436

2. Ben-Ami R, Weinberger M, Orni-Wasserlauff R, et al. Time to blood culture positivity as a marker for catheter-related candidemia. J Clin Microbiol. 2008;46(7):2222-2226.

doi:10.1128/JCM.00214-08

3. Lortholary O, Renaudat C, Sitbon K, et al. The risk and clinical outcome of candidemia depending on underlying malignancy. Intensive Care Med. 2017;43(5):652-662. doi:10.1007/s00134-017-4743-y

4. Pappas PG, Kauffman CA, Andes DR, et al. Executive Summary: Clinical Practice Guideline for the Management of Candidiasis: 2016 Update by the Infectious Diseases Society of America. Clin Infect Dis. 2016;62(4):409-417. doi:10.1093/cid/civ1194

5. Maertens J, Marchetti O, Herbrecht R, et al. European guidelines for antifungal management in leukemia and hematopoietic stem cell transplant recipients: summary of the ECIL 3--2009 update. Bone Marrow Transplant. 2011;46(5):709-718. doi:10.1038/bmt.2010.175 6. Ullmann AJ, Akova M, Herbrecht R, et al. ESCMID* guideline for the diagnosis and management of Candida diseases 2012: adults with haematological malignancies and after haematopoietic stem cell transplantation (HCT). Clin Microbiol Infect. 2012;18 Suppl 7:53-67. doi:10.1111/1469-0691.12041

7. Andes DR, Safdar N, Baddley JW, et al. Impact of treatment strategy on outcomes in patients with candidemia and other forms of invasive candidiasis: a patient-level quantitative review of randomized trials. Clin Infect Dis. 2012;54(8):1110-1122. doi:10.1093/cid/cis021 8. Nucci M, Anaissie E, Betts RF, et al. Early removal of central venous catheter in patients with candidemia does not improve outcome: analysis of 842 patients from 2 randomized clinical trials. Clin Infect Dis. 2010;51(3):295-303. doi:10.1086/653935

9. Mermel LA, Allon M, Bouza E, et al. Clinical practice guidelines for the diagnosis and management of intravascular catheter-related infection: 2009 Update by the Infectious Diseases Society of America. Clin Infect Dis. 2009;49(1):1-45. doi:10.1086/599376

10. Blot F, Nitenberg G, Chachaty E, et al. Diagnosis of catheter-related bacteraemia: a prospective comparison of the time to positivity of hub-blood versus peripheral-blood cultures. Lancet. 1999;354(9184):1071-1077. doi:10.1016/s0140-6736(98)11134-0 
11. Raad I, Hanna H, Maki D. Intravascular catheter-related infections: advances in diagnosis, prevention, and management. Lancet Infect Dis. 2007;7(10):645-657. doi:10.1016/S14733099(07)70235-9

12. Bouza E, Alcalá L, Muñoz P, et al. Can microbiologists help to assess catheter involvement in candidaemic patients before removal? Clin Microbiol Infect. 2013;19(2):E129135. doi:10.1111/1469-0691.12096

13. Park K-H, Lee MS, Lee S-O, et al. Diagnostic usefulness of differential time to positivity for catheter-related candidemia. J Clin Microbiol. 2014;52(7):2566-2572.

doi:10.1128/JCM.00605-14

14. Fernández-Cruz A, Martín-Rabadán P, Suárez-Salas M, et al. Is it feasible to diagnose catheter-related candidemia without catheter withdrawal? Med Mycol. 2014;52(5):491-497. doi:10.1093/mmy/myu013

15. Huang L, Zhang YY, Sun LY. Time to positivity of blood culture can predict different Candida species instead of pathogen concentration in candidemia. Eur J Clin Microbiol Infect Dis. 2013;32(7):917-922. doi:10.1007/s10096-013-1826-8

16. Cobos-Trigueros N, Kaasch AJ, Soriano A, et al. Time to positivity and detection of growth in anaerobic blood culture vials predict the presence of Candida glabrata in candidemia: a two-center European cohort study. J Clin Microbiol. 2014;52(8):3082-3084.

doi:10.1128/JCM.01198-14

17. Paugam A, Ancelle T, Lortholary O, Bretagne S, French Mycosis Study Group. Longer incubation times for yeast fungemia: importance for presumptive treatment. Diagn Microbiol Infect Dis. 2014;80(2):119-121. doi:10.1016/j.diagmicrobio.2014.05.013

18. Dudoignon E, Alanio A, Anstey J, et al. Outcome and potentially modifiable risk factors for candidemia in critically ill burns patients: A matched cohort study. Mycoses. 2019;62(3):237246. doi: $10.1111 /$ myc. 12872

19. Bretagne S, Renaudat C, Desnos-Ollivier M, et al. Predisposing factors and outcome of uncommon yeast species-related fungaemia based on an exhaustive surveillance programme (2002-14). J Antimicrob Chemother. 2017;72(6):1784-1793. doi:10.1093/jac/dkx045

20. Brun-Buisson C, Abrouk F, Legrand P, Huet Y, Larabi S, Rapin M. Diagnosis of central venous catheter-related sepsis. Critical level of quantitative tip cultures. Arch Intern Med. 1987;147(5):873-877. 
21. Mellinghoff SC, Cornely OA, Jung N. Essentials in Candida bloodstream infection. Infection. 2018;46(6):897-899. doi:10.1007/s15010-018-1218-1

22. Maki DG, Weise CE, Sarafin HW. A semiquantitative culture method for identifying intravenous-catheter-related infection. N Engl J Med. 1977;296(23):1305-1309.

doi:10.1056/NEJM197706092962301

23. Kollef M, Micek S, Hampton N, Doherty JA, Kumar A. Septic shock attributed to Candida infection: importance of empiric therapy and source control. Clin Infect Dis. 2012;54(12):17391746. doi:10.1093/cid/cis305

24. Buss BA, Baures TJ, Yoo M, et al. Impact of a Multiplex PCR Assay for Bloodstream Infections With and Without Antimicrobial Stewardship Intervention at a Cancer Hospital. Open Forum Infect Dis. 2018;5(10):ofy258. doi:10.1093/ofid/ofy258

25. Pulcrano G, Iula DV, Vollaro A, et al. Rapid and reliable MALDI-TOF mass spectrometry identification of Candida non-albicans isolates from bloodstream infections. J Microbiol Methods. 2013;94(3):262-266. doi:10.1016/j.mimet.2013.07.001

26. Klingspor L, Lindbäck E, Ullberg M, Özenci V. Seven years of clinical experience with the Yeast Traffic Light PNA FISH: Assay performance and possible implications on antifungal therapy. Mycoses. 2018;61(3):179-185. doi:10.1111/myc.12722

27. Kim S-H, Yoon YK, Kim MJ, Sohn JW. Clinical impact of time to positivity for Candida species on mortality in patients with candidaemia. J Antimicrob Chemother. 2013;68(12):28902897. doi:10.1093/jac/dkt256

28. Nunes CZ, Marra AR, Edmond MB, da Silva Victor E, Pereira CAP. Time to blood culture positivity as a predictor of clinical outcome in patients with Candida albicans bloodstream infection. BMC Infect Dis. 2013;13:486. doi:10.1186/1471-2334-13-486

29. Bouza E, Alvarado N, Alcalá L, et al. A prospective, randomized, and comparative study of 3 different methods for the diagnosis of intravascular catheter colonization. Clin Infect Dis. 2005;40(8):1096-1100. doi:10.1086/428576

30. De Almeida BM, Breda GL, Queiroz-Telles F, Tuon FF. Positive tip culture with Candida and negative blood culture: to treat or not to treat? A systematic review with meta-analysis. Scand J Infect Dis. 2014;46(12):854-861. doi:10.3109/00365548.2014.952246

31. Sherertz RJ, Raad II, Belani A, et al. Three-year experience with sonicated vascular catheter cultures in a clinical microbiology laboratory. J Clin Microbiol. 1990;28(1):76-82. 
32. Renz N, Cabric S, Morgenstern C, Schuetz MA, Trampuz A. Value of PCR in sonication fluid for the diagnosis of orthopedic hardware-associated infections: Has the molecular era arrived? Injury. 2018;49(4):806-811. doi:10.1016/j.injury.2018.02.018

33. Jones RL, Sayles HR, Fey PD, Rupp ME. Effect of Clinical Variables on the Volume of Blood Collected for Blood Cultures in an Adult Patient Population. Infect Control Hosp Epidemiol. 2017;38(12):1493-1497. doi:10.1017/ice.2017.230

34. Janapatla RP, Yan J-J, Chien M-L, Chen H-M, Wu H-M, Wu J-J. Effect of overnight storage of blood culture bottles on bacterial detection time in the BACTEC 9240 blood culture system. J Microbiol Immunol Infect. 2010;43(2):126-132. doi:10.1016/S1684-1182(10)60020-5

35. Clancy CJ, Pappas PG, Vazquez J, et al. Detecting Infections Rapidly and Easily for Candidemia Trial, Part 2 (DIRECT2): A Prospective, Multicenter Study of the T2Candida Panel. Clin Infect Dis. 2018;66(11):1678-1686. doi:10.1093/cid/cix1095

36. White PL, Barnes RA, Gorton R, Cruciani M, Loeffler J, Fungal PCR Initiative (Working Group of the International Society of Human and Animal Mycology). Comment on: T2Candida MR as a predictor of outcome in patients with suspected invasive candidiasis starting empirical antifungal treatment: a prospective pilot study. J Antimicrob Chemother. 2019;74(2):532-533. doi:10.1093/jac/dky325 
Table 1: Main characteristics of the 105 patients selected for the central venous catheter (CVC)related yeast fungaemia study.

\begin{tabular}{|c|c|c|c|}
\hline & $\begin{array}{l}\text { Patients with } \\
\text { CVC culture } \\
n=105\end{array}$ & $\begin{array}{l}\text { Non-CVC-related } \\
\text { yeast fungaemia } \\
\qquad \mathbf{n}=73\end{array}$ & $\begin{array}{c}\text { CVC-related } \\
\text { yeast fungaemia } \\
\qquad \mathbf{n}=32\end{array}$ \\
\hline Age median (years) [range] & $56[15-91]$ & $53[15-82]$ & $63[18-91]$ \\
\hline Sex ratio $M / F$ & 1.9 & 1.8 & 2 \\
\hline \multicolumn{4}{|l|}{ Underlying condition, n (\%) } \\
\hline Oncology & $90(86)$ & $63(86)$ & $27(84)$ \\
\hline Acute leukaemia & $36(40)$ & $28(44)$ & $8(29.6)$ \\
\hline $\begin{array}{l}\text { Chronic lymphoproliferative } \\
\text { disorders }\end{array}$ & $32(36)$ & $27(43)$ & $5(18.5)$ \\
\hline Solid tumour & $22(24)$ & $8(13)$ & $14(52)$ \\
\hline No malignancy & $5(4)$ & $3(4)$ & $2(6)$ \\
\hline Solid organ transplantation & $4(80)$ & $2(67)$ & $2(100)$ \\
\hline HIV positivity & $1(20)$ & $1(33)$ & 0 \\
\hline Not specified & $10(10)$ & $7(10)$ & $3(9)$ \\
\hline ICU, n (\%) & $39(37)$ & $29(40)$ & $10(33)$ \\
\hline Antifungal pre-exposure, n (\%) & $24(23)$ & $22(30)^{a}$ & $2(6)^{a}$ \\
\hline Recent surgery, n (\%) & $21(20)$ & $12(16)$ & $9(28)$ \\
\hline $\begin{array}{l}\text { Long-term central venous access } \\
\text { catheter, } n(\%)\end{array}$ & $82(78)$ & $55(75)$ & $27(84)$ \\
\hline $\begin{array}{l}\text { Median number of positive } \\
\text { BCs/patient [IQR] }\end{array}$ & $2[1-5]$ & $2[1-4]$ & $4[2-5]$ \\
\hline Mortality within 30 days, n (\%) & $\begin{array}{c}\text { 30/103 (29) } \\
2 \mathrm{LFU}\end{array}$ & $20 / 73(27)$ & $\begin{array}{c}10 / 30(33) \\
2 \mathrm{LFU}\end{array}$ \\
\hline Yeast species n (\%) & & & \\
\hline Candida albicans & $48(46)$ & $35(48)$ & $13(41)$ \\
\hline C. parapsilosis & $20(19)$ & $15(21)$ & $5(16)$ \\
\hline
\end{tabular}




\begin{tabular}{lccc}
\hline C. glabrata & $16(15)$ & $9(12)$ & $7(22)$ \\
C. tropicalis $_{\text {C. Iusitaniae }}$ & $9(9)$ & $6(8)$ & $3(9)$ \\
Others $^{\text {b }}$ & $2(2)$ & $1(1)$ & $1(3)$ \\
\hline
\end{tabular}

CVC: Central Venous Catheter, M/F: Male/Female Ratio, HIV: Human Immuno-deficiency Virus, ICU: Intensive Care Unit, BC: Blood Culture. LFU: Lost of Follow Up

${ }^{\mathrm{a}} \mathrm{p}=0.007$ (Chi ${ }^{2}$ test) between non CVC-related fungaemia and CVC-related fungaemia

b Others: only one episode per yeast species: Candida dubliniensis, Candida fabianii, Candida inconspicua, Candida kefyr (Kluveromyces marxianus), Candida krusei, Candida palmioleophila, Geotrichum clavatum, Rhodotorula mucilaginosa, Saccharomyces cerevisiae, Trichosporon inkin.

Table 2: Performance of the different cut-offs for TTP to predict CVC-related candida infection

\begin{tabular}{|c|c|c|c|c|c|c|}
\hline $\begin{array}{c}\text { Cut-off } \\
\text { (time in } \\
\text { hours) }\end{array}$ & $\begin{array}{c}\text { Sensitivity } \\
\% \text { [95\% CI] }\end{array}$ & $\begin{array}{c}\text { Specificity } \\
\%[95 \% \mathrm{CI}]\end{array}$ & $\begin{array}{c}\text { Positive } \\
\text { likelihood } \\
\text { ratio }\end{array}$ & $\begin{array}{c}\text { Negative } \\
\text { likelihood } \\
\text { ratio }\end{array}$ & $\begin{array}{c}\text { Positive } \\
\text { predictive } \\
\text { value (\%) }\end{array}$ & $\begin{array}{c}\text { Negative } \\
\text { predictive } \\
\text { value (\%) }\end{array}$ \\
\hline$<5$ & $\begin{array}{c}9.4 \\
{[2-25]}\end{array}$ & $\begin{array}{c}98.7 \\
{[92-99]}\end{array}$ & 7.2 & 0.9 & 75.0 & 71.8 \\
\hline$<10$ & $\begin{array}{c}28.1 \\
{[13-46]}\end{array}$ & $\begin{array}{c}98.7 \\
{[92-99]}\end{array}$ & 21.6 & 0.7 & 90.0 & 76.3 \\
\hline$<15$ & $\begin{array}{c}46.9 \\
{[29-65]}\end{array}$ & $\begin{array}{c}90.7 \\
{[81-96]}\end{array}$ & 5.0 & 0.6 & 68.2 & 80.0 \\
\hline$<20$ & $\begin{array}{c}59.4 \\
{[40-76]}\end{array}$ & $\begin{array}{c}80.0 \\
{[69-88]}\end{array}$ & 3.0 & 0.5 & 55.9 & 82.2 \\
\hline$<25$ & $\begin{array}{c}71.9 \\
{[53-86]}\end{array}$ & $\begin{array}{c}66.7 \\
{[54-77]}\end{array}$ & 2.2 & 0.4 & 47.9 & 84.7 \\
\hline$<30$ & $\begin{array}{c}75.0 \\
{[56-88]}\end{array}$ & $\begin{array}{c}49.3 \\
{[37-61]}\end{array}$ & 1.5 & 0.5 & 38.7 & 82.2 \\
\hline$<35$ & $\begin{array}{c}78.1 \\
{[60-90]}\end{array}$ & 34.7 & 1.2 & 0.6 & 33.8 & 78.8 \\
\hline
\end{tabular}




\begin{tabular}{|c|c|c|c|c|c|c|}
\cline { 5 - 7 }$<40$ & $\begin{array}{c}90.6 \\
{[74-98]}\end{array}$ & $\begin{array}{c}28.0 \\
{[18-39]}\end{array}$ & 1.3 & 0.3 & 34.9 & 87.5 \\
\hline$<45$ & $\begin{array}{c}90.6 \\
{[74-98]}\end{array}$ & $\begin{array}{c}18.7 \\
{[11-30]}\end{array}$ & 1.1 & 0.5 & 32.2 & 82.4 \\
\hline$<50$ & $\begin{array}{c}93.8 \\
{[79-99]}\end{array}$ & $\begin{array}{c}13.3 \\
{[6-23]}\end{array}$ & 1.1 & 0.5 & 31.6 & 83.3 \\
\hline$<55$ & $\begin{array}{c}93.8 \\
<79-99]\end{array}$ & $\begin{array}{c}9.3 \\
<3-18]\end{array}$ & 1.0 & 0.7 & 30.6 & 77.8 \\
\hline
\end{tabular}

TTP: time to positivity, DTTP: differential time to positivity; CI: confidence interval 
Table 3: Performance of the different DTTP cut-off values and their validity to predict catheterrelated Candida infection

\begin{tabular}{|c|c|c|c|c|c|c|}
\hline $\begin{array}{l}\text { Cut-off } \\
\text { (time in } \\
\text { hours) }\end{array}$ & $\begin{array}{l}\text { Sensitivity } \\
\%[95 \% \mathrm{CI}]\end{array}$ & $\begin{array}{l}\text { Specificity } \\
\%[95 \% \mathrm{CI}]\end{array}$ & $\begin{array}{c}\text { Positive } \\
\text { likelihood } \\
\text { ratio }\end{array}$ & $\begin{array}{c}\text { Negative } \\
\text { likelihood } \\
\text { ratio }\end{array}$ & $\begin{array}{l}\text { Positive } \\
\text { predictive } \\
\text { value (\%) }\end{array}$ & $\begin{array}{l}\text { Negative } \\
\text { predictive } \\
\text { value (\%) }\end{array}$ \\
\hline$>1$ & $\begin{array}{c}100.0 \\
{[80-100]}\end{array}$ & $\begin{array}{c}61.5 \\
{[40-79]}\end{array}$ & 2.6 & 0.0 & 63.0 & 100.0 \\
\hline$>2$ & $\begin{array}{c}100.0 \\
{[80-100]}\end{array}$ & $\begin{array}{c}69.2 \\
{[48-85]}\end{array}$ & 3.2 & 0.0 & 68.0 & 100.0 \\
\hline$>3$ & $\begin{array}{c}100.0 \\
{[80-100]}\end{array}$ & $\begin{array}{c}70.8 \\
{[50-87]}\end{array}$ & 3.4 & 0.0 & 70.8 & 100.0 \\
\hline$>4$ & $\begin{array}{c}100.0 \\
{[80-100]}\end{array}$ & $\begin{array}{c}70.8 \\
{[52-88]}\end{array}$ & 3.4 & 0.0 & 70.8 & 100.0 \\
\hline$>5$ & $\begin{array}{c}100.0 \\
{[80-100]}\end{array}$ & $\begin{array}{c}70.8 \\
{[53-90]}\end{array}$ & 3.4 & 0.0 & 70.8 & 100.0 \\
\hline$>6$ & $\begin{array}{c}94.1 \\
{[71-99]}\end{array}$ & $\begin{array}{c}75.0 \\
{[56-91]}\end{array}$ & 3.8 & 0.1 & 72.7 & 94.7 \\
\hline$>10$ & $\begin{array}{c}82.4 \\
{[56-96]}\end{array}$ & $\begin{array}{c}79.2 \\
{[60-93]}\end{array}$ & 4.0 & 0.2 & 73.7 & 86.4 \\
\hline$>15$ & $\begin{array}{c}76.5 \\
{[43-90]}\end{array}$ & $\begin{array}{c}79.2 \\
{[60-93]}\end{array}$ & 3.7 & 0.3 & 72.2 & 82.6 \\
\hline$>18$ & $\begin{array}{c}60.0 \\
{[37-84]}\end{array}$ & $\begin{array}{c}87.5 \\
{[65-95]}\end{array}$ & 4.8 & 0.5 & 75.0 & 77.8 \\
\hline
\end{tabular}

DTTP: differential time to positivity 


\section{Legends to Figures:}

Figure 1: Flow Chart of the study proces

TTP: time to positivity, DTTP: differential time to positivity. Paired bottles collected within 30 minutes. *Exclusion criteria: lack of CVC Culture, lack of Blood Culture through CVC, coinfection with bacteria, mixed yeasts infection.

Figure 2: Comparisons of time to positivity (TTP) between positive (CVC+) and negative (CVC-) central venous catheter for all yeast cultured and according to the four more frequent Candida spp. $(*: p=0.0001)$. Data presented in scatter dot-plot with the median line and the interquartile range.

Figure 3: Receiver operating characteristic (ROC) curve analysis of time to positivity (TTP) for all yeasts (blue line; AUC $=0.73$ ), C. albicans (green line; AUC $=0.84$ ), and non-albicans Candida spp. (pink line; AUC = 0.64).

Figure 4: Receiver operating characteristic (ROC) curve analysis of differential time to positivity $(\mathrm{DTTP})$ for all yeasts (blue line; AUC = 0.86), C. albicans (green line; AUC =0.84), non-albicans Candida spp. (pink line; AUC $=0.90)$. 


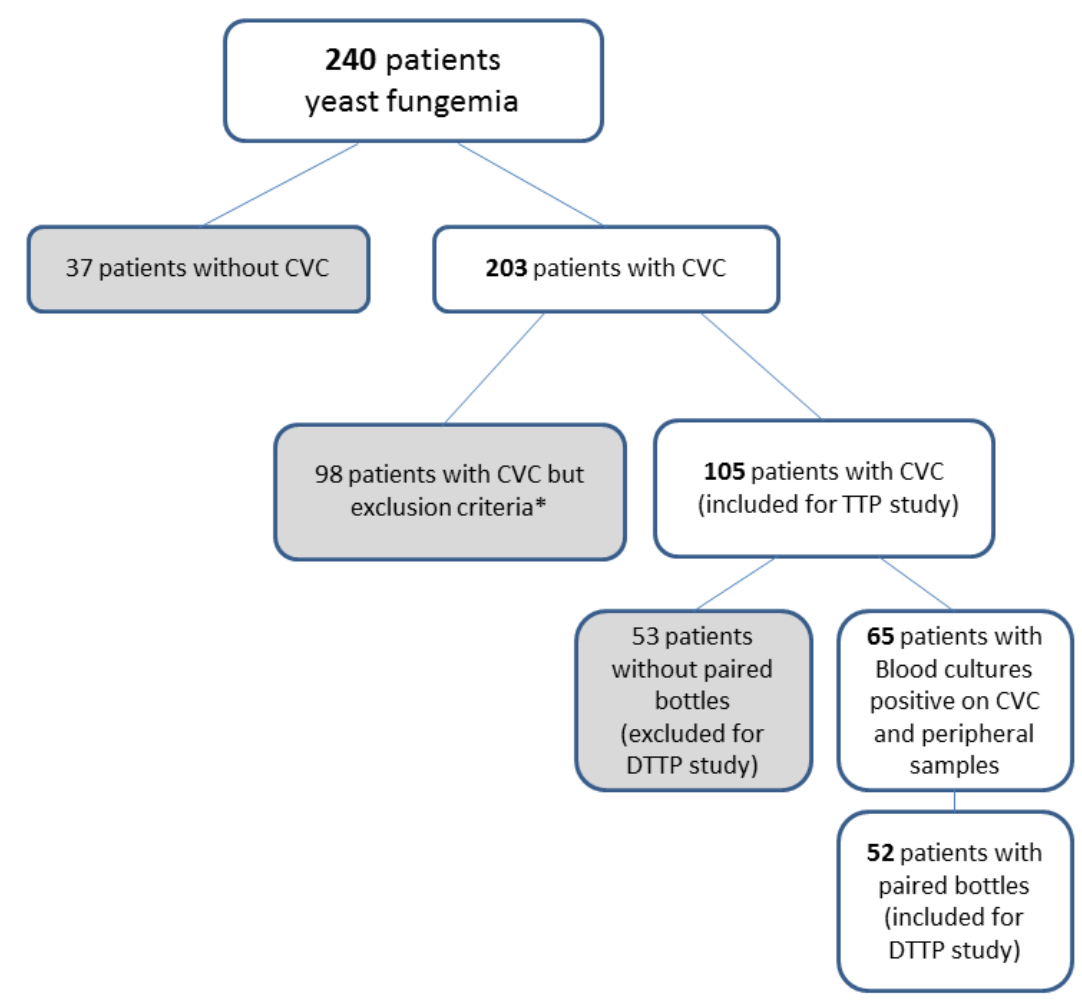

myc_13024_f1.tif 


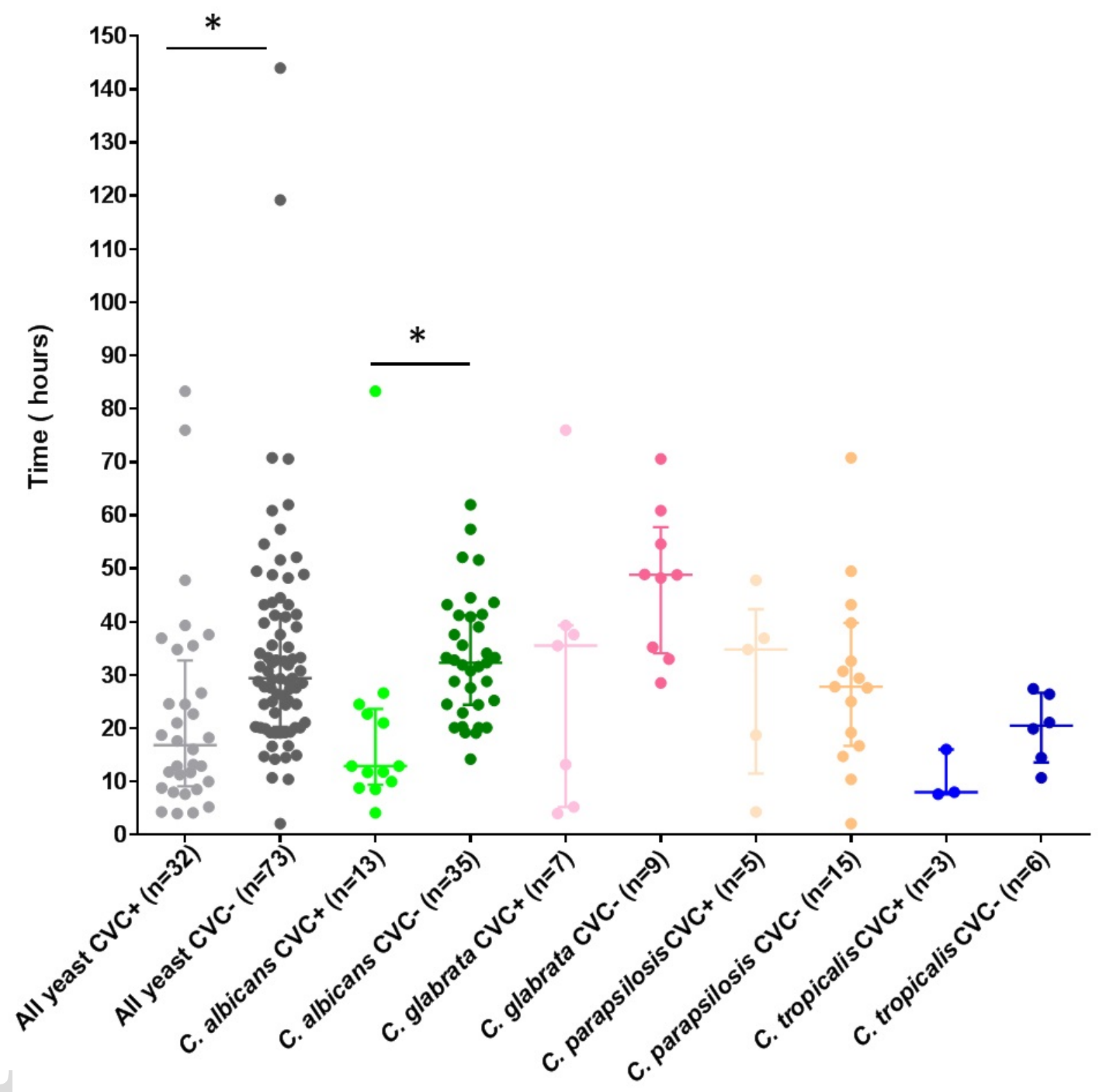

myc_13024_f2.jpg 


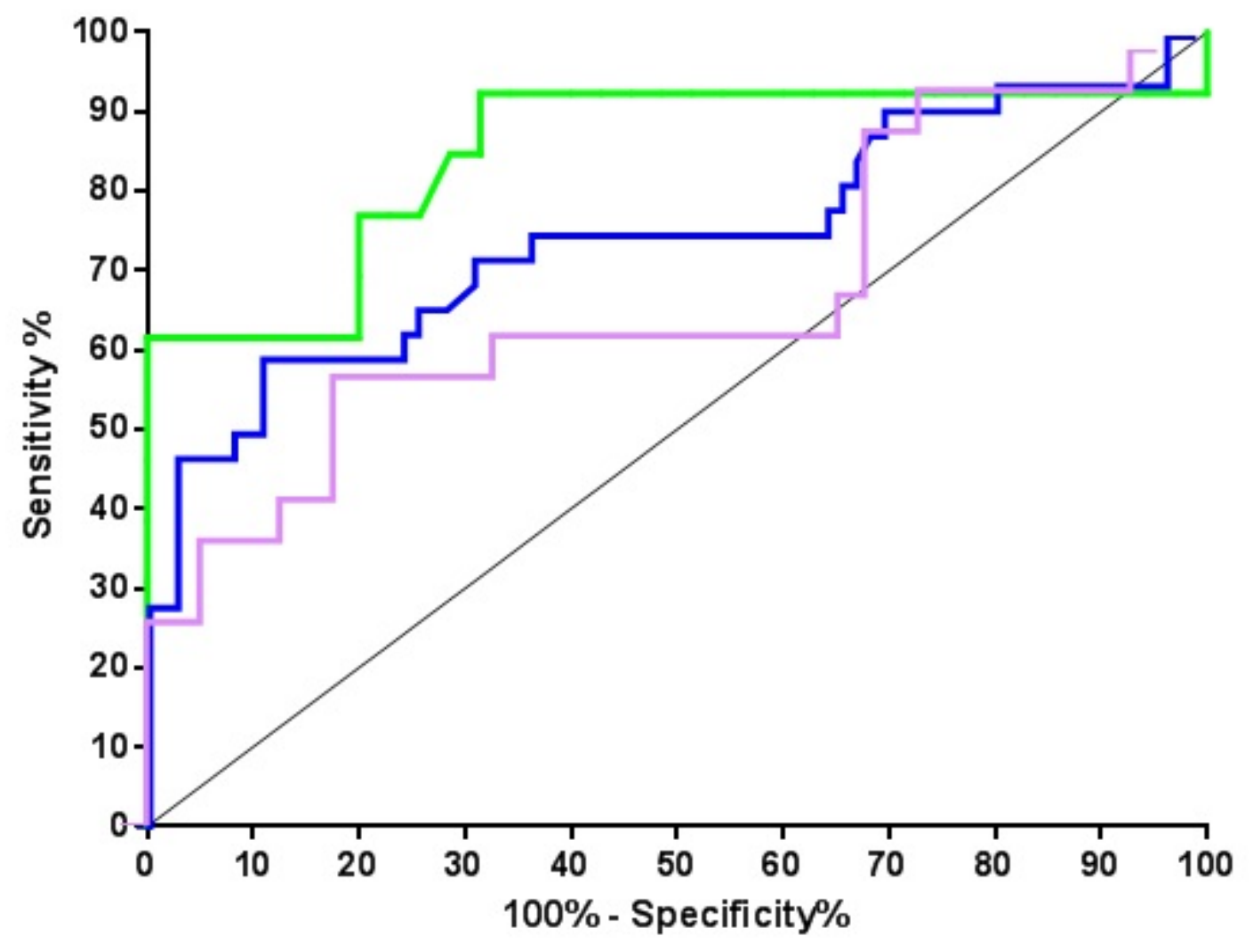

myc_13024_f3.tif 


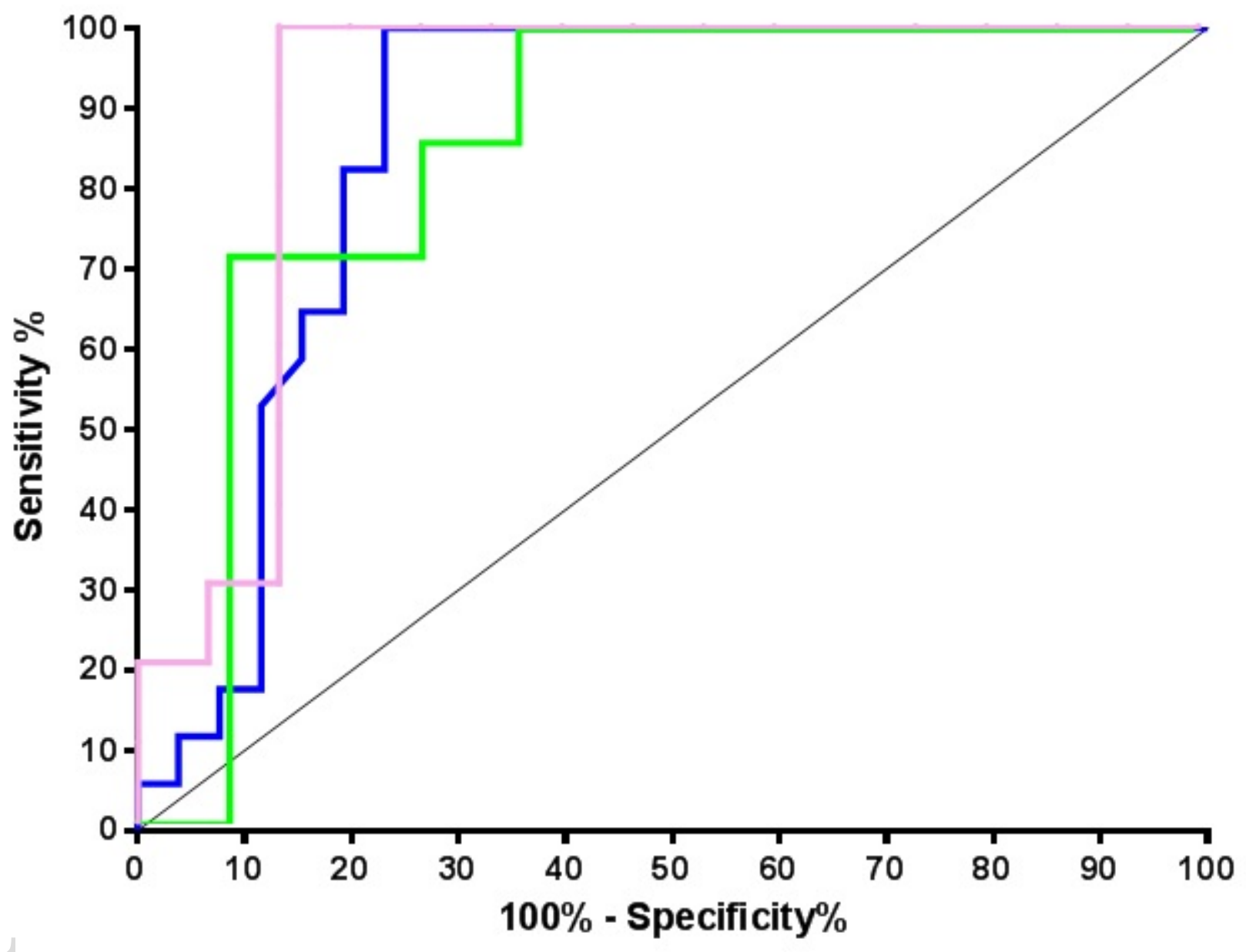

myc_13024_f4.tif 